\title{
INFLUENCE OF GAS-FILLED GAPS ON THE THERMAL BEHAVIOUR OF DUAL PURPOSE CASKS
}

\author{
Dinkel, Christian (1); Billenstein, Daniel (1); Rieg, Frank (1); Roith, Bernd (2) \\ 1: University of Bayreuth; 2: Swiss Federal Nuclear Safety Inspectorate ENSI
}

\begin{abstract}
Basically, the safe dissipation of heat is among others an important protection objective of dual purpose casks. Gas-filled gaps within such casks can play a major role for the thermal behavior as they act as thermal barriers due to the lower heat conductivity of gaseous fluids in comparison to metallic materials. However, additional heat transmission mechanisms, such as natural convection and radiation can also occur in a gaseous medium. This leads to both an expanded modelling and a prolonged computing time in numerical simulations. Within the scope of a research project in cooperation with Swiss Federal Nuclear Safety Inspectorate ENSI a simulation tool for the fast thermal evaluation of dual purpose casks is developed which combines analytical methods and FEA. The innovation is that the thermal effects of gas-filled gaps are considered by using analytical equations. Main focus lies on the implementation of heat radiation as a non-linear transfer mechanism. Therefore, an iterative calculation process is used and the effects of the iteration number is investigated. Furthermore, the influence of radiation in comparison to pure conduction is examined depending on the gap width.
\end{abstract}

Keywords: Simulation, Product modelling / models, Numerical methods, Thermal Finite-Element Analysis, Dual purpose cask

\section{Contact:}

Dinkel, Christian

University of Bayreuth

Chair of Engineering Design and CAD

Germany

christian.dinkel@uni-bayreuth.de 


\section{INTRODUCTION}

Dual purpose casks (DPC) for nuclear fuel elements have to fulfil several protection objectives, according to the guideline TS-R-1 (IAEA, 2005), in order to ensure the safe transport (first purpose) and storage (second purpose) of the nuclear material. Basically, these protection objectives can be summarized as follows (IAEA, 2005): containment of the radioactive contents, control of external radiation levels, prevention of criticality and prevention of damage caused by heat.

In order to ensure the strict compliance with each objective during an approval procedure, dual purpose casks have to proof safety according to special safety cases like drop tests and fire tests. Therefore, numerical methods are more and more important in the framework of the cask development next to experimental verification processes to meet the high requirements, which are mandatory during the approval procedure.

In case of the protection objective 'prevention of damage caused by heat', the cask has to be able to safely dissipate the decay heat, which is still generated by the fuel assemblies during transport and storage without the use of external cooling systems. In order to ensure the stability and mechanical integrity of the cask, the maximum temperature is limited on certain positions and for certain parts, due to the fact that the strength of materials decreases as temperature loads increase. At the same time, thermal stresses due to different thermal expansion occur.

Basically, the fuel elements within dual purpose casks are positioned in the basket, which represents the inner cask part (Droste et al., 2014). This part is surrounded by a cylindrical shell, which ensures subcriticality due to the use of neutron-absorbing materials (e.g. resins) and high mechanical strength. At the top, a dual lid system seals the cask, whereas a plate is usually welded to the cask at its bottom (Droste et al., 2014), (Ziegler and Allelein, 2013). A loaded cask is about six meters in length, has a diameter of about 2.5 to 3 meters and a weight of about 140 tons (Ziegler and Allelein, 2013). An exemplary and simplified cask model is shown in Figure 1. In case of the HI-STAR 180 cask designed by Holtec International, the temperature is limited for the cladding of the fuel elements $\left(314{ }^{\circ} \mathrm{C}\right)$, for the basket $\left(275^{\circ} \mathrm{C}\right)$, for the cask surface $\left(118^{\circ} \mathrm{C}\right)$ and many more positions, such as seals etc. (Holtec International, n.d.).

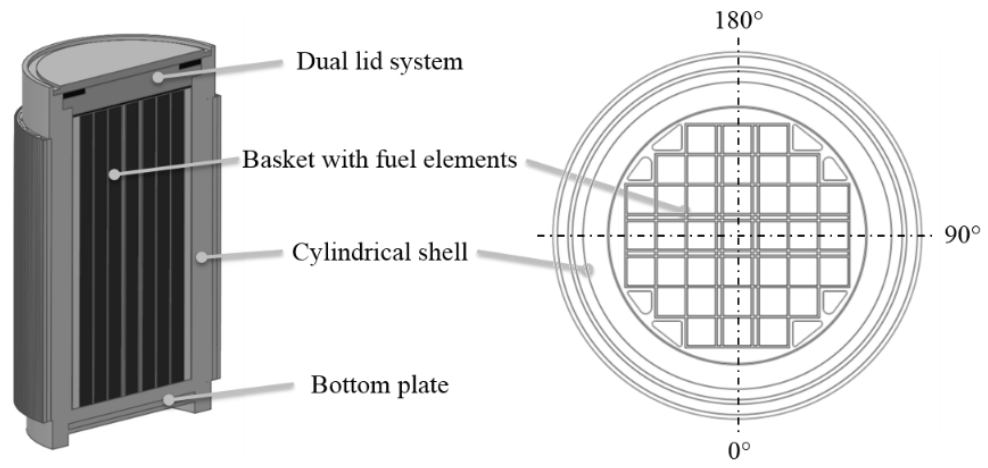

Figure 1. Isometric (left) and cross-sectional (right) view of a simplified cask model including lid system, basket, cylindrical shell (outer cask part) and bottom plate

A particular challenge for efficient numerical analysis regarding the thermal behaviour of DPC poses gas-filled gaps. For an inert environment and a high thermal conductivity, DPC are evacuated after loading and filled with Helium afterwards. Since not only heat conduction exists as heat transfer mechanism in fluids, but convection and heat radiation occur as well, the consideration of gas-filled gaps within numerical simulations leads to a higher computational effort as well as to an increased modelling expense, which is why such gaps are often neglected or modelled as solid material with pure conduction. Additionally, such gaps usually show very small geometrical dimensions in comparison to the overall cask dimensions, which makes them even more difficult to consider within thermal analyses. One regarded gas-filled gap, which is particularly important for the heat dissipation in radial direction, is positioned between basket and cavity wall. Normally, a basic assumption is a perfectly centered basket for an upright positioned cask, which leads to an annular gap geometry, as Figure 2 shows. For conservative reasons, this is a safe assumption, as maximum temperatures are calculated (Koch et al., 2007). 


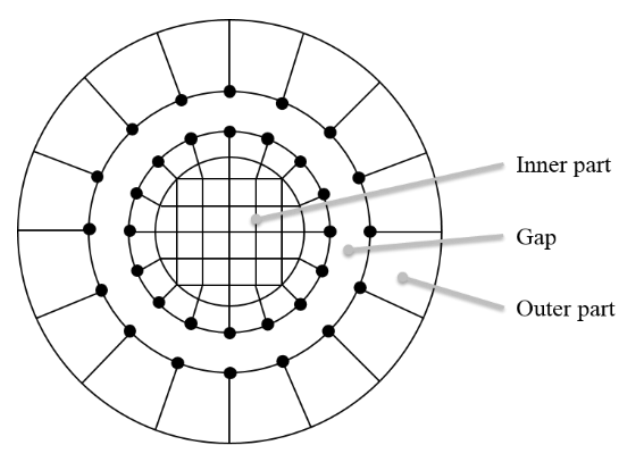

Figure 2. Schematic finite-element illustration of the annular gap geometry

Due to the low thermal conductivity of fluids in comparison to metallic solids and due to the fact that convection and radiation exist as further heat transfer mechanisms in fluids next to conduction, such gas-filled gaps are more complicated to simulate than pure solid components.

Within the scope of two research projects a newly simulation tool for the fast thermal evaluation of dual purpose casks was developed in cooperation with the Swiss Federal Nuclear Safety Inspectorate ENSI, which combines analytical methods and Finite-Element-Analysis (FEA). The innovation thereby is that gas-filled gaps are not meshed. Their influence on thermal processes is rather considered by using analytical equations. By means of special mathematical algorithms, additional functions between nodes can be applied, even though these nodes are not connected by a finite element. These functions act similar to constraints and are referred to as thermal gap conditions (TGC). By the use of TGC, significant advantages like a decreased modelling effort and reduced computing time can be achieved.

Here, the method of implementing thermal gap conditions is developed for but not limited to DPC. The better understanding of the thermal behaviour and the effects of thermal expansion can be crucial for many products as thermal stresses can occur and place additional burdens on the strength of materials. Therefore, gap conditions can be used to simulate the temperature distribution more accurate, whenever the basic geometry of the considered gap is simple enough to calculate it by analytical equations, for example in the case of double glazed windows or heat pipe isolations. So, a more accurate knowledge of the temperature field can be provided.

\section{DEVELOPMENT OF A SIMULATION TOOL FOR THE THERMAL ASSESSMENT OF DUAL PURPOSE CASKS}

\subsection{Initial situation and objectives}

Numerical simulations are a crucial part for the development of DPC. At the same time, extensive simulations with a high modelling depth are very time consuming both during product development in case of product designers and during approval in case of the approval authority. For the latter, a simulation tool (ST) was developed and is subject to continuous further development, which allows the fast thermal evaluation of DPC for arbitrary boundary conditions and gap sizes, whereas the tool is highly automated to ensure an easy and fast use. At the same time, the ST enables the user to consider all kinds of heat transfer mechanisms, which would usually require Computational Fluid Dynamicssimulations (CFD). Due to the fact, that CFD-simulations solve not only the temperature field, which is required to know for the mentioned protection objective, but also the flow field (in case of free convection), the computational effort is much higher.

Furthermore, the ST considers the thermal behaviour of gas-filled gaps automatically. The increase in calculation speed is achieved by an analytical consideration of fluid regions, whereas solid cask regions are solved using conventional thermal FEA. Both analytical and numerical methods are combined for a fast simulation. A first version of this ST was introduced at the International Symposium on the Packaging and Transportation of Radioactive Materials (Dinkel et al., 2016) in 2016. For this, radiation as a non-linear heat transfer mechanism (see Equation 1) was neglected and solely conduction and convection was implemented. For verification purposes a test bench was build and the functioning of the simulation tool could be verified successfully, see Chapter 2.4.

The basic physical correlation of radiation is given in Equation 1. For a heat transfer $\dot{Q}$ caused by radiation, $C_{12}$ symbolizes the radiation exchange number, which is defined by the emissivity 
coefficients of the participating surfaces and the Boltzmann constant, $A$ represents the surface and $T_{1}$ and $T_{2}$ the temperature values (Incropera et al., 2007).

$$
\dot{Q}=C_{12} A\left(T_{1}^{4}-T_{2}^{4}\right)
$$

As Equation 1 shows, heat transfer by radiation is non-linear, which makes it more complicated to consider within simulations. Furthermore, Equation 1 indicates that the influence of radiation gets bigger with an increasing gap width, as in this case the temperature difference at the gap rises and a greater amount of heat can be transported by radiation.

Despite the non-linearity, a new version of the ST is presented here, which includes radiation as a further heat transfer mechanisms within fluids analytically. At first, the functioning of the simulation tool in combination with the thermal gap condition is discussed in Chapter 2.2 to show the implementation of radiation together with conduction and convection on an analytical basis. After that, the influence of radiation on the thermal behaviour of dual purpose casks is analysed in Chapter 2.3.

\subsection{Thermal gap condition}

In order to bridge unmeshed areas within Finite-Element-Analysis, a special mathematical function needs to connect the divided parts in the absence of finite elements in this area. The basic principle is illustrated in Figure 3. It can be seen, that both basket (inner cask part) and cylindrical shell (outer cask part) are meshed using Finite-Element principles. Both parts are divided by an annular gap in case of upright positioned casks (see also Figure 2). The gap is equipped with a gap condition, which connects inner and outer parts (see dashed lines in Figure 3).

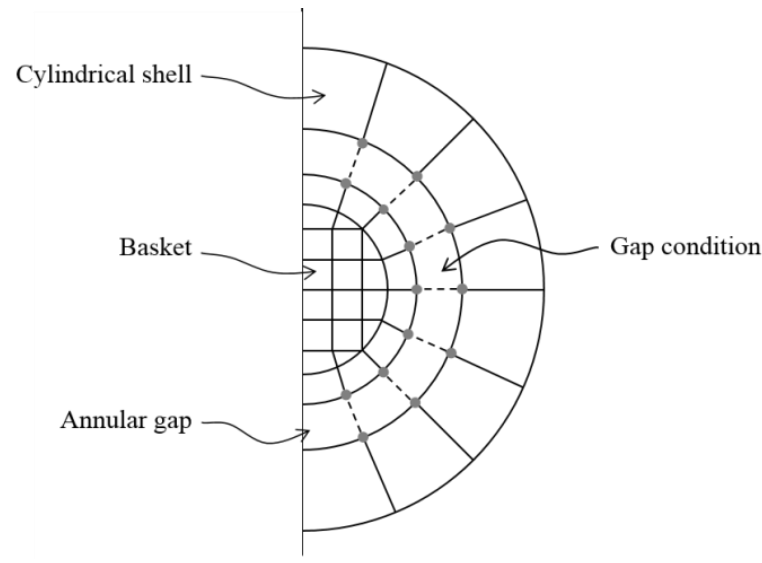

Figure 3. Illustration of the thermal gap condition for bridging the unmeshed area of an annular gas-filled gap

Therefore, a modified form of the perturbed Lagrange method is used (Cook et al., 2002). Frequently, the perturbed Lagrange or perturbed Lagrange multiplier method is applied for solving contact simulations, which means, that this method is able to connect divided parts by inserting additional equations in the mathematical equation system of FEA (Rust, 2011). Basically, the ST performs a conventional thermal FE-simulation. Therefore, the conductivity matrices of inner and outer cask part are compiled and the boundary conditions are inserted in the numerical system of equations. After this is done, the gap conditions are implemented by adding additional rows and columns to the equational system. This can be shown by using an easy example of two thermal truss elements divided by a gap, see Figure 4, wherein the nodes number 2 and number 3 have to be connected. As gap condition, it is defined that the temperature values at node number 2 and 3 are equal.

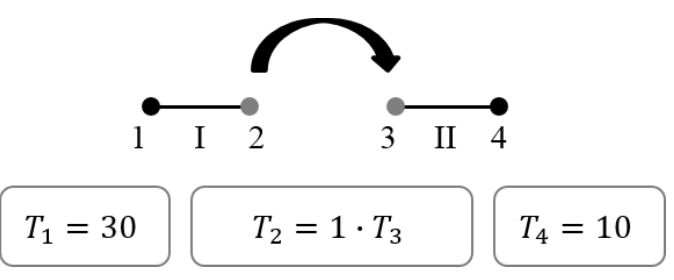

Figure 4. Minimal example of two trusses divided by a gap for the fundamental functioning of the perturbed Lagrange method 
By considering the exemplary boundary conditions in Figure 4 (temperature value of 30 for node number 1 and temperature value of 10 for node number 4 ), the temperature solution for node 2 and 3 , on condition that the same element geometry and material properties are used for both truss elements, has to be of the value 20 , as far as the gap condition is satisfied. This solution is also successfully calculated by the simulation tool.

As stated before, bridging the gap can be reached by inserting additional equations to the equation system. This is done by adding one additional column and row for each node pair which should be connected, as Equation 2 states for instance.

In Equation 2, the basic equation system for thermal FEA is shown, which solves the unknown temperature vector $\boldsymbol{T}$ with the help of the conductivity matrix $\boldsymbol{K}$ and the heat flow vector $\boldsymbol{Q}$. Within matrix $K, K_{I}^{e}$ and $K_{I I}^{e}$ represent the element conductivity values for element I and II. In case of truss elements, which are not connected, the first four rows and columns of matrix $\boldsymbol{K}$ would describe the unconnected system.

$$
\boldsymbol{K} \boldsymbol{T}=\left[\begin{array}{ccccc}
K_{I}^{e} & -K_{I}^{e} & 0 & 0 & 0 \\
-K_{I}^{e} & K_{I}^{e} & 0 & 0 & 1 \\
0 & 0 & K_{I I}^{e} & -K_{e}^{I I} & -1 \\
0 & 0 & -K_{I I}^{e} & K_{I I}^{e} & 0 \\
0 & 1 & -1 & 0 & -\frac{1}{\alpha}
\end{array}\right]\left(\begin{array}{c}
T_{1} \\
T_{2} \\
T_{3} \\
T_{4} \\
\lambda_{p L}
\end{array}\right)=\left(\begin{array}{c}
Q_{1} \\
Q_{2} \\
Q_{3} \\
Q_{4} \\
0
\end{array}\right)=\boldsymbol{Q}
$$

The fifth row and column shows the functioning of the perturbed Lagrange method and introduces an additional equation in order to connect the gap nodes of the minimal example. By the use of the factors 1 and -1 , the demanded gap condition is fulfilled, as the same temperature value is required for the nodes 2 and 3. In case of the perturbed Lagrange method, the penalty factor $\alpha$ on the fifth diagonal element in matrix $\boldsymbol{K}$ is used to improve the numerical solvability of the system. For deeper information, see (Cook et al., 2002) and (Zienkiewicz et al., 2013). Summarized, it can be said, that by this method linear, analytical equations can be inserted in FEA.

\subsubsection{Conduction and convection}

As stated before, a first version of the simulation tool considered only conduction and convection as heat transfer mechanisms in fluids, whereby radiation was neglected. Therefore, the basic gap condition equations are given in this chapter, whereas the extension of the TGC involving radiation is described in Chapter 2.2.2.

In case of conduction and an annular gap geometry, an analytical equation describes the influence of the gasfilled gap depending on the temperature values at the gap, see Equation 3, generally named $T_{O P}$ (index OP: outer part) and $T_{I P}$ (index IP: inner part) (Dinkel et al., 2016), (VDI, 2013):

$$
T_{O P}-T_{I P}=-\frac{\dot{Q}}{2 \pi l \lambda_{F l}} \ln \left(\frac{r_{O P}}{r_{I P}}\right)
$$

Here, $\lambda_{F l}$ marks the thermal conductivity of the fluid in the gap, which usually is Helium for dual purpose casks. Additionally, $l$ stands for the gap width, $\dot{Q}$ for the heat flow from inner to outer part and $r_{O P}$ and $r_{I P}$ for the radius of inner and outer part.

For the consideration of convection, the fluid conductivity value is manipulated by the use of the Nusselt number $\mathrm{Nu}$, which is a dimensionless number for the existence of natural convection (VDI, 2013). Therefore, the Nusselt number is multiplied with the former value of the fluid conductivity $\lambda_{F l}$ and a new, fictive conductivity $\lambda_{C C}$ is implemented in the gap condition function shown in Equation 3 (index CC: conduction and convection):

$$
\lambda_{C C}=N u \cdot \lambda_{F l}
$$

To do so, the Nusselt number is calculated automatically by the simulation tool using empirical equations of Müller (Müller, 1999). For Nusselt numbers larger than 1, equation 4 will be applicable, if not, the value of $\mathrm{Nu}$ is set to 1 , which means that no natural convection exists within the gap. No user interaction is needed for the correct choose of the thermal gap condition. 


\subsubsection{Radiation}

As for the perturbed Lagrange method only linear equations are possible to consider, radiation cannot be implemented in its original form which was shown in Equation 1. Therefore, radiation has to be linearized for the use as gap condition. This can be done in a similar way as convection is considered as well. According to VDI Wärmeatlas (VDI, 2013), a fictive heat transfer coefficient for radiation can be calculated, if the heat transfer mechanisms are considered as being independent from each other, as the following equation shows:

$$
\lambda_{R}=4 l C_{12} T_{m}^{3}
$$

Herein, $T_{m}$ represents a mean temperature value for one node pair, where a gap condition has to be equipped. The remaining parameters have already been explained. Using this newly calculated heat transfer coefficient for radiation, it can be added to the combined conductivity of conduction and convection $\lambda_{C C}$, whereby again a new conductivity value for the fluid in the gap is calculated (VDI, 2013):

$$
\lambda_{R C C}=\lambda_{C C}+\lambda_{R}
$$

This new value $\lambda_{R C C}$ (Index RCC: radiation, conduction and convection) can be implemented in the basic gap function of pure conduction shown in Equation 3. Doing this, the final gap function, which is integrated using the perturbed Lagrange method, is still a linear equation. But as Equation 5 states, a mean temperature must be known for the calculation of the heat transfer coefficient for radiation $\lambda_{R}$, which is not possible as the temperature in the gap is not known unless the system is solved. Therefore, an iterative approach has to be chosen. For this reason, the new simulation tool uses for a first iteration pure conduction as heat transfer mechanism and solves the temperature distribution in the cask. This way, the temperature values for the gap nodes are already known. Now, this results can be used to calculate the mean temperature $T_{m}$, based on pure conduction. For a second iteration, $T_{m}$ owns an initial value and radiation is considered as well for the TGC. Therefore, it has to be investigated, how an appropriate value for the amount of iterations can be found. This is done in Chapter 2.3.

A further challenge is the required calculation of view factors. View factors are defined as the proportion of a radiation heat flow emitted from surface $A_{1}$ that hits surface $A_{2}$. For this reason, the view factor $F_{1 \rightarrow 2}$ between two random surfaces $A_{1}$ and $A_{2}$ with the Euclidean distance $s$ can be calculated by (VDI, 2013):

$$
F_{1 \rightarrow 2}=\frac{1}{\pi A_{1}} \int_{A_{2}} \int_{A_{1}} \frac{\cos \beta_{1} \cos \beta_{2}}{s^{2}} d A_{1} d A_{2}
$$

The two angles $\beta_{1}$ and $\beta_{2}$ describe the respective angle between the surface normal vectors and the connecting line between both surfaces, as given in Figure 5 (VDI, 2013):

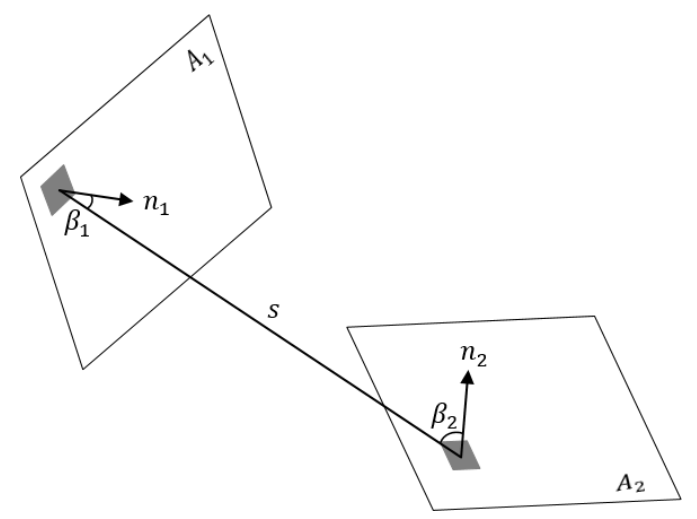

Figure 5. General calculation of the view factor between two random surfaces following (Incropera et al., 2007)

View factors have to be included for calculations, if only a part of the heat flow through radiation emitted by a surface can hit another surface. For this case, Equation 1 is extended (VDI, 2013):

$$
\dot{Q}=F_{12} C_{12} A_{1}\left(T_{1}^{4}-T_{2}^{4}\right)
$$


For this reason, the calculation effort for FEA simulation considering radiation - even if it is linearized increases due to the fact, that the geometry of the participating parts have to be checked for their view factors. In case of the developed simulation tool, this was done both for an annular and a crescent gap geometry, whereby only annular gaps are evaluated within the frame of this paper. Because the use of radiation means an increase of computational effort, it can be disabled within the simulation tool for a faster but more inexact calculation.

\subsection{Exemplary cask simulations}

Using exemplary cask simulations, the influence of radiation on the total heat dissipation can be seen. As many design information of DPC are confidential, the cask model derives from public available information only. It follows the HI-STAR 180 DPC, which is designed by Holtec International, in a simplified way, as only basic geometrical dimensions are publically available (Holtec International, n.d.). On this basis, the cask model is reduced to three components: basket (conductivity $\lambda_{B}=200 \mathrm{~W} / \mathrm{mK}$ ), basket attachment $\left(\lambda_{B A}=50 \mathrm{~W} / \mathrm{mK}\right)$ and cylindrical shell $\left(\lambda_{C S}=8 \mathrm{~W} / \mathrm{mK}\right)$. The lid system as well as the bottom plate is neglected within these analyses. In the following, upright positioned casks with an annular gap geometry are evaluated in terms of the proportion of radiation on the heat dissipation and in terms of the numerical stability of the implemented iterative calculation of radiation. Therefore, Figure 6 shows the used cask model. It contains heat sources of $31.76 \mathrm{~kW}$ in total, which are distributed over the several fuel element slots (see arrows in Figure 6) according to a HI-STAR 180 loading plan (Holtec International, n.d.). On the outer cask surface a convection boundary condition is applied using a heat transfer coefficient $\alpha=8 \mathrm{~W} / \mathrm{m}^{2} \mathrm{~K}$ and an ambient temperature $T_{\infty}=20^{\circ} \mathrm{C}$ (see dashed line around the cask in Figure 6). All attached boundary conditions are distributed evenly over the height of the cask. The FE-model consists in total of 430,680 degrees of freedom and 334,224 linear hexahedron elements (basket: 212,652 elements; basket attachment: 98,064; cylindrical shell: 23,508), which are capable of performing thermal and thermo-mechanical simulations. As the used model shows a rotational symmetry regarding both the boundary conditions and the mesh, the resulting temperature distribution is also expected to be rotationally symmetric.

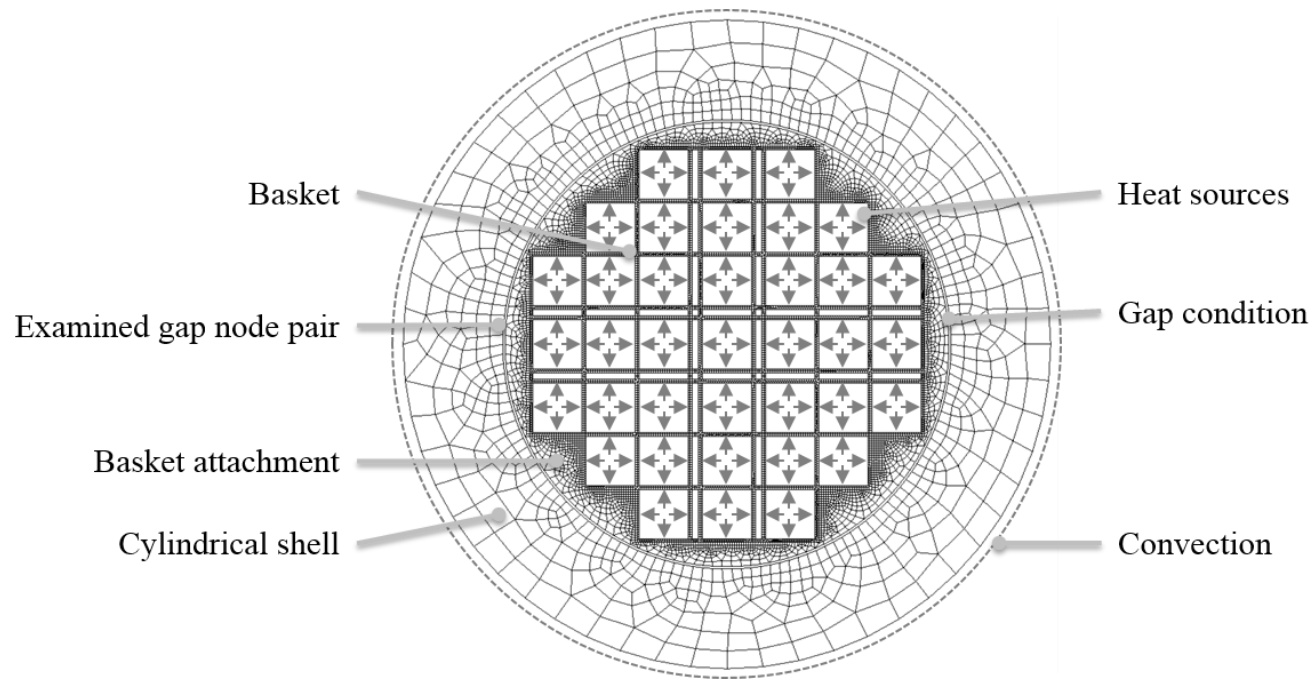

Figure 6. FE-model of the exemplary cask including component names, boundary conditions (heat source on each basket wall and convection boundary condition at outer cask surface) and the location of an examined gap node pair

At first, the influence of the iteration amount is evaluated, as the implementation of radiation entails an iterative solution process. Therefore, both the maximum cask temperature $T_{\max }$ and the nodal temperature of one exemplary node pair ( $T_{\text {node }, I P}$ and $T_{\text {node, } O P}$, see Figure 6 for nodal position) is compared and displayed in Table 1. A gap width of $10 \mathrm{~mm}$ is used for this evaluation. 
Table 1. Influence of the iteration number on the maximum cask temperature and on one gap node pair in ${ }^{\circ} \mathrm{C}$

\begin{tabular}{|c|c|c|c|c|c|}
\hline $\begin{array}{c}\text { Iteration } \\
\text { number }\end{array}$ & 2 & 4 & 6 & 8 & 10 \\
\hline$T_{\max }$ & 288.9627 & 289.9825 & 289.9846 & 289.9846 & 289.9846 \\
\hline$T_{\text {node }, I P}$ & 260.1907 & 261.2100 & 261.2120 & 261.2120 & 261.2120 \\
\hline$T_{\text {node }, O P}$ & 217.4769 & 217.4795 & 217.4795 & 217.4795 & 217.4795 \\
\hline
\end{tabular}

It can be seen, that there is very little influence of the iteration number for the implementation of radiation on the maximum temperature and as well on the nodal temperature of one node pair, which is connected by a thermal gap condition. For an iteration number of 4 , no more changes exist in comparison to an iteration number of 10. The difference between the iteration numbers 2 and 4 is about 1 Kelvin maximum, wherefore in the following simulations only two iterations are used as long as radiation is considered in order to achieve a low simulation time. This means, that the consideration of radiation does not change the temperature of the gap nodes very much, because the conductivity $\lambda_{C C}$ is significantly bigger than $\lambda_{R}$ for this example (see Equation 6), which leads to a good convergence.

The marginal deviations between the different iteration numbers can also be seen in Figure 7. Here, the temperature distribution for iteration number 2 (Figure 7, left) and iteration number 10 (Figure 7, right) is given. For both temperature distributions, the scale is unified. It therefore appears, that the choice of an iteration number of 2 is sufficiently accurate in case of this simulation tool as not only the maximum cask temperature diverge only very little, but also the qualitative temperature distribution matches.
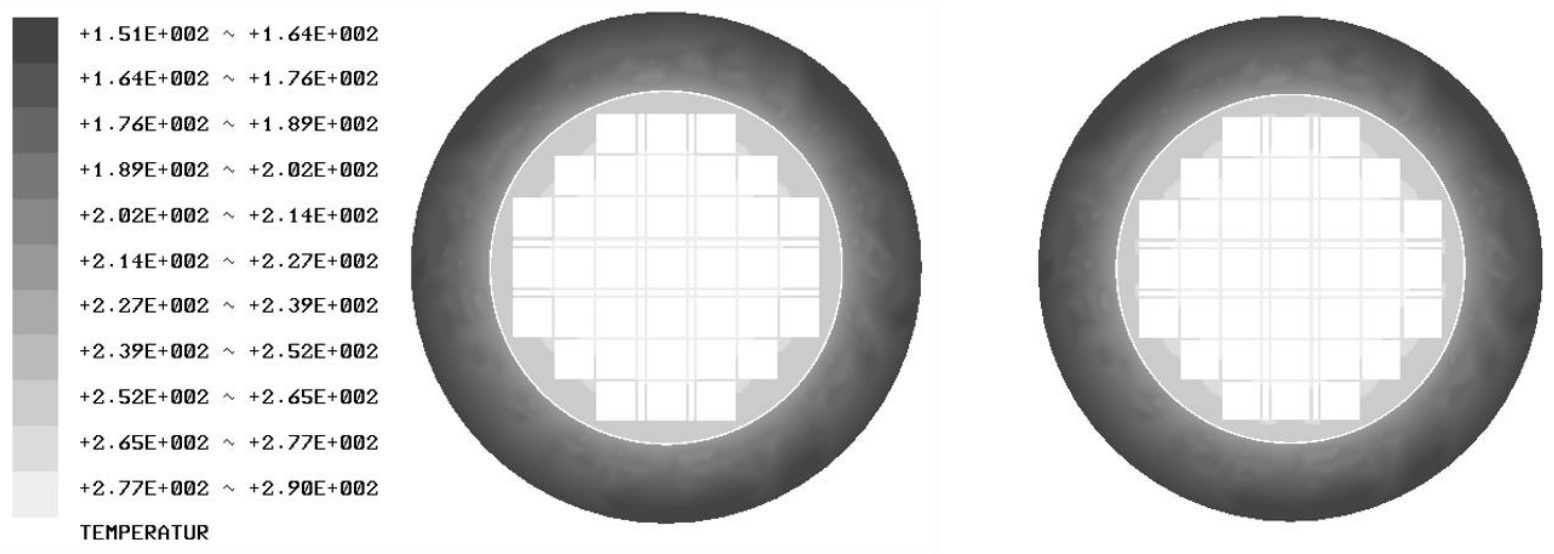

\section{Figure 7. Comparison of the temperature distribution in ${ }^{\circ} \mathrm{C}$ for the iteration number 2 (left) and 10 (right)}

Subsequently, the next step is the quantification of radiation as a heat transfer mechanism itself in comparison to pure conduction. This is done in combination with a variation of the gap width $(2,4$, 6,8 and $10 \mathrm{~mm}$ ), because the real gap width during operation is unknown due to thermal expansion and production tolerances. As criteria, the maximum cask temperature is compared $\left(T_{\max , C}\right.$ : pure conduction; $T_{\max , R C}$ : radiation plus conduction). Therefore, the same Finite-Element model is used, except the gap width is adapted each time. Table 2 shows the maximum temperature of each simulation together with the deviation between the single heat transfer mechanisms.

Table 2. Influence of radiation and gap width on the maximum temperature in ${ }^{\circ} \mathrm{C}$

\begin{tabular}{|c|c|c|c|c|c|}
\hline Gap width & $2 \mathrm{~mm}$ & $4 \mathrm{~mm}$ & $6 \mathrm{~mm}$ & $8 \mathrm{~mm}$ & $10 \mathrm{~mm}$ \\
\hline$T_{\max , C}$ & 260.41 & 273.57 & 286.73 & 299.89 & 313.05 \\
\hline$T_{\max , R C}$ & 259.23 & 269.09 & 277.16 & 286.71 & 288.96 \\
\hline Deviation & $0.46 \%$ & $1.66 \%$ & $3.45 \%$ & $4.59 \%$ & $8.34 \%$ \\
\hline
\end{tabular}

These results illustrate that the influence of radiation is higher, the bigger the gap width is. At the same time, the deviation between pure conduction and the combined consideration of conduction and radiation is not bigger than $8.34 \%$ for this exemplary simulation. For instance, the temperature distribution for two gap 
widths is given in Figure 8 (left: $10 \mathrm{~mm}$, right: $2 \mathrm{~mm}$ ) by considering both conduction and radiation as gap condition. Here, the temperature distribution is significantly influenced by the assumed gap width. For both temperature distributions, the scale is unified. Because of the small geometrical dimension, the gap width of $2 \mathrm{~mm}$ (Figure 8, right) can hardly be detected.

Summarized it can be said, that for more exact results, radiation should be applied as a gap condition, but may be neglected, if a very fast simulation speed is aimed. Furthermore, the non-consideration of radiation always produces conservative temperature results, as the maximum temperature is higher for conduction only.
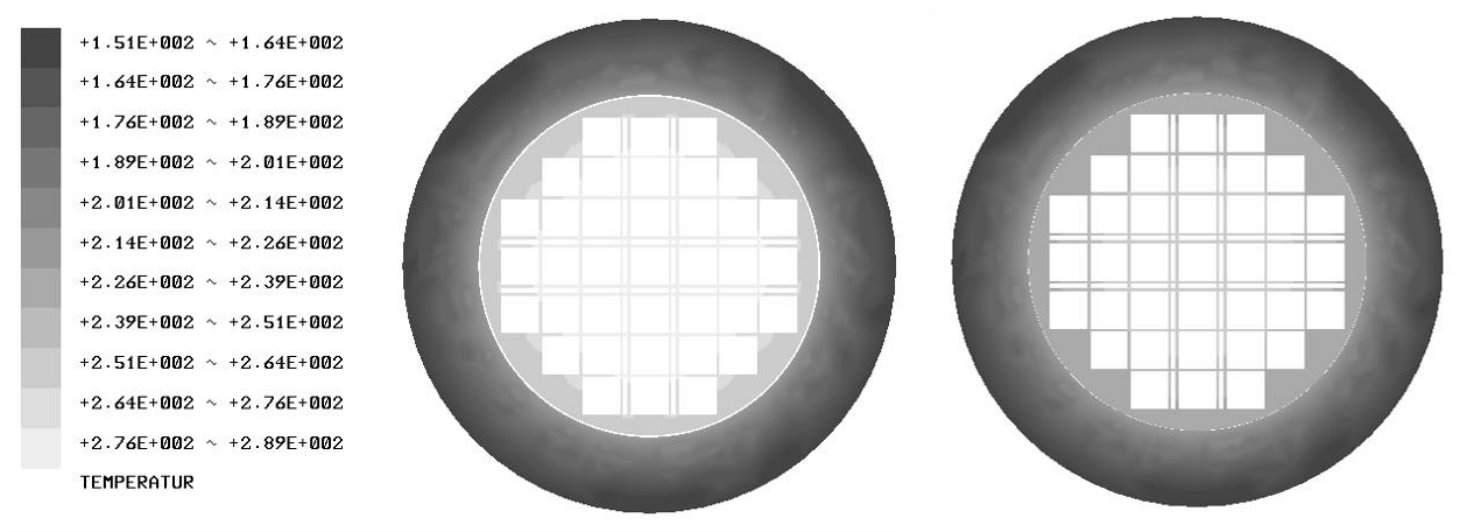

Figure 8. Comparison of the temperature distribution in ${ }^{\circ} \mathrm{C}$ for the gap width of $10 \mathrm{~mm}$ (left) and $2 \mathrm{~mm}$ (right)

\subsection{Test bench comparison}

In order to validate the thermal gap condition for combined heat transfer mechanisms, a test bench was designed, which represents a downscaled (scale approximately 1:10) and simplified DPC. The gap geometry itself is not downscaled. The test bench owns an annular gap of $10 \mathrm{~mm}$ width. Basically, the test bench consists of two concentric cylinders, which build the gap geometry. At the top, a lid system closes the test bench, whereas a plate is welded to the bottom. In the centre, a heating tape is able to heat up the test bench and the fluid in the inside up to $200{ }^{\circ} \mathrm{C}$. For a better accordance with reality, the test bench is evacuated and filled with Helium afterwards, which means increased leak tightness requirements. Thermocouples are equipped at and in the gap to monitor the temperature distribution at several positions. The basic test bench components are modelled for a FE-simulation using the newly developed ST including thermal gap conditions for conduction, convection and radiation. Two temperature levels are compared between measurement and simulation: $200{ }^{\circ} \mathrm{C}$ and $150{ }^{\circ} \mathrm{C}$. These values mean that the temperature at the inner gap wall is $200{ }^{\circ} \mathrm{C}$ or $150{ }^{\circ} \mathrm{C}$. As boundary conditions, a convective heat transfer is defined for the outer test bench wall (heat transfer coefficient $\alpha=8 \mathrm{~W} / \mathrm{m}^{2} \mathrm{~K}$, ambient temperature $T_{\infty}=23^{\circ} \mathrm{C}$ ), whereas a heat flow of $280 \mathrm{~W}$ (temperature level $200{ }^{\circ} \mathrm{C}$ ) respectively $195 \mathrm{~W}$ (temperature level $150^{\circ} \mathrm{C}$ ) represents the heating tape of the test bench. Each measurement takes place when the heating phase is over and a stationary temperature distribution can be seen. Both temperature levels are measured four times, whereas the temperature values are averaged. Table 3 shows the results of the comparison of test bench (Index TB) and simulation values (Index ST) regarding the temperature difference in the gap for the temperature level of $200{ }^{\circ} \mathrm{C}$, measured at three positions (position $0^{\circ}, 90^{\circ}$ and $180^{\circ}$, see Figure 1 ) along the circumference of the gap.

Table 3: Comparison of measured temperature values at the gap and simulated results for the temperature level of $200^{\circ} \mathrm{C}$

\begin{tabular}{|c|c|c|c|}
\hline & Position $0^{\circ}$ & Position $90^{\circ}$ & Position $180^{\circ}$ \\
\hline$\Delta T_{G a p, S T}$ in $\mathrm{K}$ & 35.75 & 35.75 & 35.75 \\
\hline$\Delta T_{G a p, T B}$ in $\mathrm{K}$ & 40.65 & 40.82 & 38.40 \\
\hline Absolute deviation in $\mathrm{K}$ & -4.90 & -5.06 & -2.65 \\
\hline Relative deviation in $\%$ & -12.05 & -12.41 & -6.90 \\
\hline
\end{tabular}

It can be seen, that the biggest absolute deviation is about $-5 \mathrm{~K}$, which is equivalent to $-12.4 \%$ relative deviation. The temperature level of $150{ }^{\circ} \mathrm{C}$, which is not shown in Table 3, provides similar results (biggest absolute deviation: $-5.01 \mathrm{~K}$ ). These deviations are considered as acceptable. 


\section{CONCLUSION AND OUTLOOK}

Thermal Finite-Element simulations of dual purpose casks are highly important for the assessment of the safe operation. Such simulations which consider gas-filled gaps, would increase the modelling and computational efforts significantly. Because of this, a simulation tool is developed which is able to provide an approximated thermal evaluation of dual purpose casks based on an analytical approach for the consideration of gas-filled gaps, which is implemented in the numerical FEA. Therefore, radiation was neglected in the first place due to its non-linear behaviour, but was now included as well as an iterative process for the thermal gap condition.

As it can be seen in this work, radiation influences the maximum temperature as the value decreases if radiation is considered as a further heat transfer mechanisms. The influence of radiation is higher, the bigger the gap width is. For the exemplary simulations presented in this paper, the deviation between pure conduction and the combined consideration of conduction and radiation is not bigger than $8.34 \%$. Furthermore, the consideration of radiation means in effect an iterative calculation process and thus an increase of calculation effort. It could be shown that an amount of two iterations is sufficiently precise in the framework of such thermal analyses of casks, as the maximum temperature value fluctuates only slightly depending on the iteration amount.

In general, the consideration of radiation leads to a better knowledge of the temperature distribution and the peak temperatures within the cask. This allows an optimized cask loading, which becomes more important in the future, as more high active fuel elements have to be stored.

Currently, further gap geometries are evaluated, which means that further thermal gap conditions have to be build. A crescent gap geometry, which can be observed in case of lying casks, is of particular interest for the future work.

\section{REFERENCES}

Cook, R., Malkus, D., Plesha, M. and Witt, R. (2002), Concepts and Applications of Finite Element Analysis, John Wiley \& Sons, Madison.

Dinkel, C., Frisch, M., Billenstein, D., Roith, B. and Rieg, F. (2016), "Development of a simulation tool for the thermal evaluation of transport and storage casks", Proceedings of the 18th International Symposium on the Packaging and Transportation of Radioactive Materials PATRAM, Kobe, 18.09.2016 - 23.09.2016.

Droste, B., Komann, S., Wille, F., Rolle, A., Probst, U. and Schubert, S. (2014), “Consideration of aging mechanism influence on transport safety of dual purpose casks for spent nuclear fuel or HLW", Packaging, Transport, Storage \& Security of Radioactive Material, Vol. 25, pp. 105-112. https://doi.org/10.1179/1746510914y.0000000070.

Holtec International (n.d.), Safety Analysis Report on the HI-STAR 180 Package, Non-proprietary Version, Report HI2073681, Revision 3, United States Nuclear Regulatory Commission, Marlton.

Incropera, F., Dewitt, D., Bergman, T. and Lavine, A. (2007), Fundamentals of Heat and Mass Transfer, John Wiley $\&$ Sons, Hoboken.

International Atomic Energy Agency IAEA (2005), Regulations for the Safe Transport of Radioactive Material: Safety Requirements No. TS-R-1, Wien.

Koch, F., Bletzer, C. and Wieser, G. (2007), "Consideration of asymmetrical heat transmission and distribution using numerical methods", Proceedings of the 15th International Symposium on the Packaging and Transportation of Radioactive Materials PATRAM, Miami, 21.10.2007 - 26.10.2007.

Müller, U. and Ehrhard, P. (1999), Freie Konvektion und Wärmeübertragung, C. F. Müller Verlag, Heidelberg.

Rust, W. (2011), Nichtlineare Finite-Elemente-Berechnungen, Vieweg+Teubner Verlag, Wiesbaden. https://doi.org/10.1007/978-3-8348-8148-9.

Verein Deutscher Ingenieure VDI (2013), VDI-Wärmeatlas, Springer Vieweg, Berlin, Heidelberg.

Ziegler, A. and Allelein, H.-J. (2013), Reaktortechnik, Springer Vieweg, Berlin, Heidelberg. https://doi.org/10.1007/978-3-642-33846-5.

Zienkiewicz, O., Taylor, R. and Zhu, J. (2013), The Finite Element Method: Its Basis and Fundamentals, Elsevier, Amsterdam, Boston, Heidelberg, London, New York, Oxford, Paris, San Diego, San Francisco, Singapore, Sydney, Tokyo.

\section{ACKNOWLEDGMENTS}

This research project was kindly supported by ENSI, Swiss Federal Nuclear Safety Inspectorate, Brugg, Switzerland. 